\title{
Atomistic Modeling of Graphene/PANI Nanocomposite
}

\author{
Zhi Guo, Minhui Pan, Miao Zhang, Ningbo Liao* \\ College of Mechanical \& Electrical Engineering, Wenzhou University, Wenzhou 325035, China.
}

1377686913@qq.com

\begin{abstract}
Understanding the structure properties of Graphene/PANI are important in developing polyaniline-based materials for different fields. In this study, the electronic property of Graphene/PANI are investigated by density functional theory. Analyses of the band structure and density of states indicated that the Graphene/PANI has excellent conductivity. By molecular dynamics caclulations, the Graphene/PANI exhibit excellent adsorption capacity towards gas molecules. These results imply that the Graphene/PANI is more favorable in detecting gas because of its high sensitivity and adsorption.
\end{abstract}

Keywords: Graphene/PANI, Nanocomposite, Electronic Property, High Sensitivity, Adsorption

\section{INTRODUCTION}

The materials play a significant role in numerous application fields such as industrial emission control, household security, medical diagnosis and environmental monitoring. The material is the heart of many hardware products and the quality of the material directly affects the performance of the products. So far, numerous researches on materials in different fields have been reported, included inorganic, inorganic oxide/dioxide and conducting polymers [1], which may be expensive, operated at high temperature or high concentrations, exhibited low response, high power consumption or low sensitivity [2]. For these reasons, nanostructured materials (nanomaterials)can also be used to reduce working temperatures and they consume less power and are safer to operate [3].

Graphene, a two-dimensional lattice of $\mathrm{C}$ atoms, has attracted a great deal of attention due to its high thermal, electrical conductivity and mechanical strength, especially its high surface-to-volume ratio [4]. Based on its structural features, graphene materials have presented a distinct gas sensing capability [5]. However, the pristine Graphene is not suitable for gas sensing applications because of low adsorption energies of test gas molecules on the GR surface [6, 7].

Hence, The incorporation of GR in polymer, graphene polymer nanocomposite is a way to get best of both materials-GR and polymer. A composite is a combination of multiple materials in which the property might be a weighted average of the components or a completely new one. Recently, Graphene/PANI nanocomposite was proposed to enhanced the sensitivity of ammonia sensor [8]. The nanocomposite Graphene/PANI films have exhibited better than pure PANI overall toluene sensing behavior in terms of sensor response, response and recovery time as well as repeatability [9]. However, So far, the theoretical study of the Graphene/PANI nanocomposite is still relatively lacking.

In this study, molecular dynamic and first-principle calculations were applied to investigate properties of Graphene/PANI nanocomposite structure. This will further guide us on find excellent performance of nanocomposite and make the application more extensive. 


\section{COMPUTATIONAL DETAILS}

MD simulations were conducted with a time step of 0.5 fs. The temperature and pressure were controlled by the Berendsen's method using a half-life for decay to the target temperature of $0.1 \mathrm{ps}$ (decay constant) and $0.1 \mathrm{ps}$ for the pressure scaling constant. The non-bonded electrostatic and van der Waals forces were controlled by Ewald method with a cutoff value of $10 \AA$. First principles calculations were used to calculated band structure and DOS of the systems. The exchange correlation interaction between electrons is described using GGA with the Perdew-Burke-Ernzerhof(PBE) [10], The core electrons were treated with DFT Ultrasoft pseudopotentials. Special points sampling integration over the Brillouin zone was employed using Monkhorst-Pack schemes with a $3 \times 3 \times 3$ k-point mesh. The cut-off energy is set to be $340 \mathrm{eV}$ for the Expansion of plane waves. All the calculations were implemented by commercial software Materials Studio.

\section{RESULT AND DISCUSSION}

Based on the characteristics of nanomaterials, it is very significant to use materials for sensors. The few sites for Graphene/PANI composite are shown in Figure. 1.(a) We consider two configurations in which the gas molecule is closed between graphene and polyaniline. Moreover for the Graphene/PANI, the gas molecule is placed on the top of bridge site at the middle of a C-C bond and the hollow site at the center of a benzene ring. The initial models for MD calculations are shown in Figure. 1.(b).

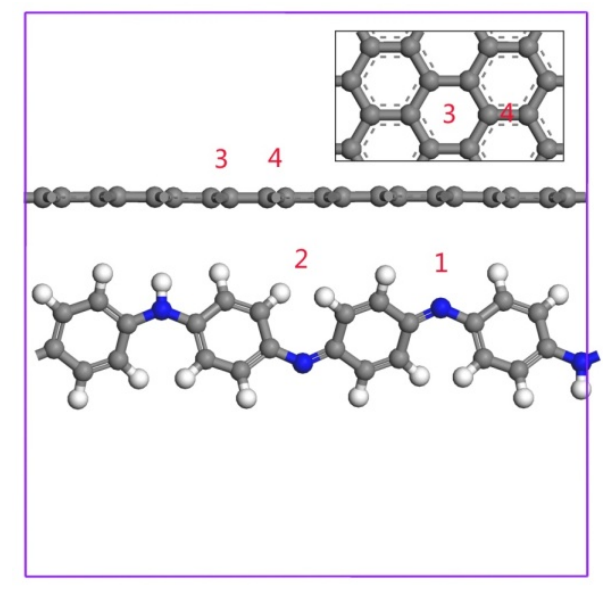

(a)

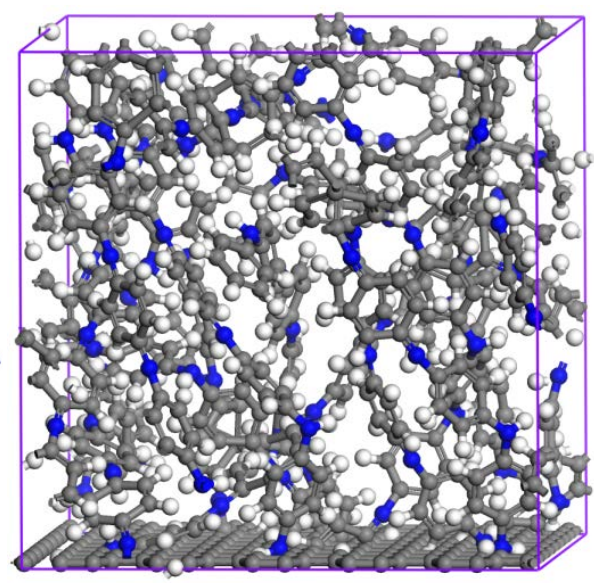

(b)

FIGURE 1.(a) The few sites on Graphene/ PANI: 1, 2, 3, 4 for QM calculation;

(b) The model of Graphene/PANI for MD calculations.

As shown in Figure 2b, the structure was stable after 3 iterations, and the energy of the equilibrium state is $-17500.099 \mathrm{eV}$. It is obvious from the Figure 2a that all the values of the results are below our set value, which 
suggest that the results are meet the computational accuracy.

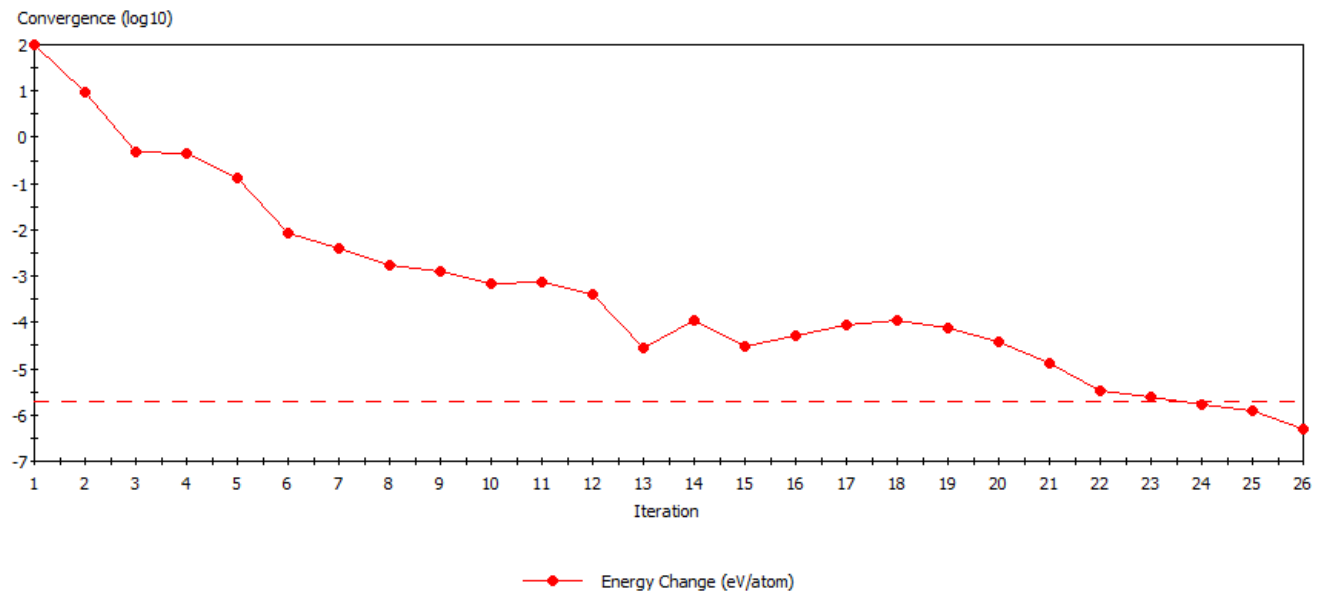

(a)

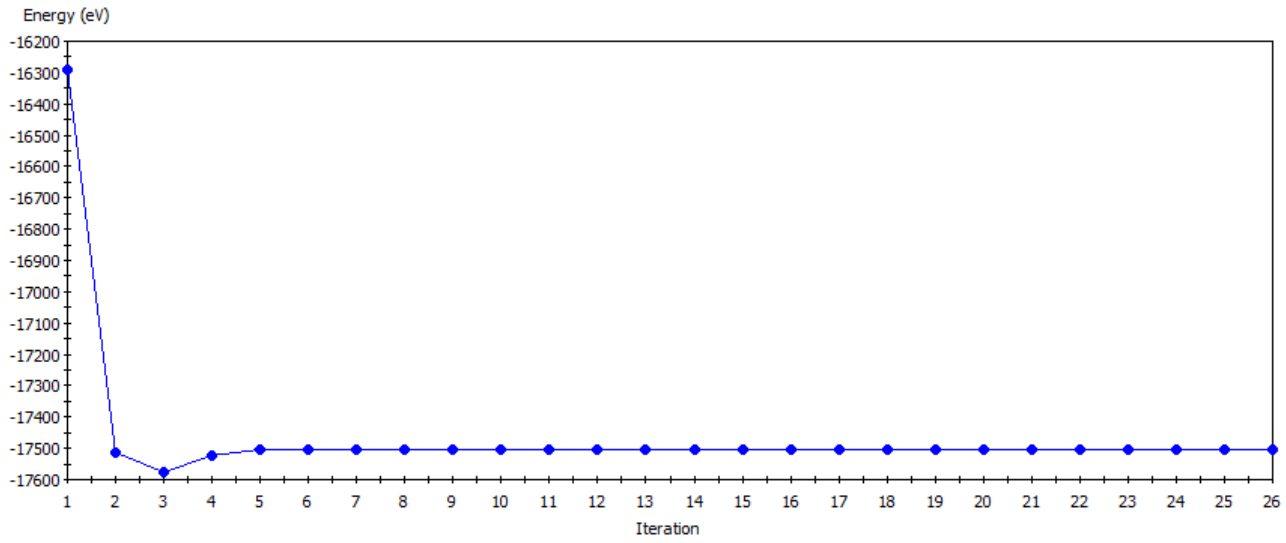

(b)

FIGURE 2.The SCF Convergence and energy geometry optimization

As can be seen from the Figure. 3, the band gap of the nanomaterials is very small, only 0.291 and the new energy level appears at the Fermi level. This indicate the Graphene/PANI has excellent conductivity and it can be used to develop sensors, so on. 
CASTEP Band Structure Band gap is $0.291 \mathrm{eV}$

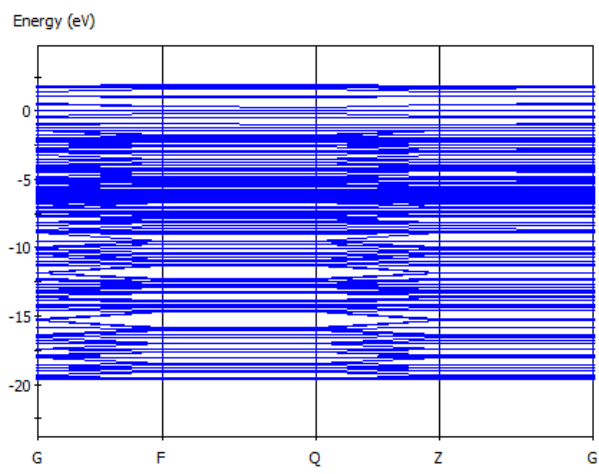

CASTEP Density of States

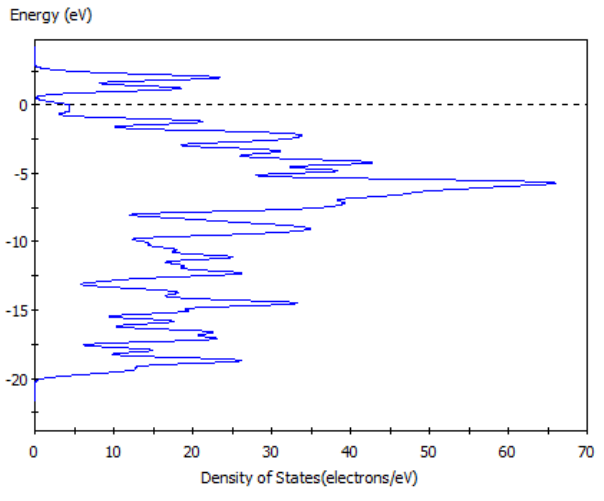

FIGURE 3.The band structure and DOS of Graphene/PANI

Further evidences for validity of our model are provided by the computed radial distribution function (RDF) of Graphene/PANI in Figure. 4. The RDF was used to analyze the spatial distribution of small molecules relative to Graphene/PANI, respectively, it can be used to study the effect of materials on the adsorption site distribution.

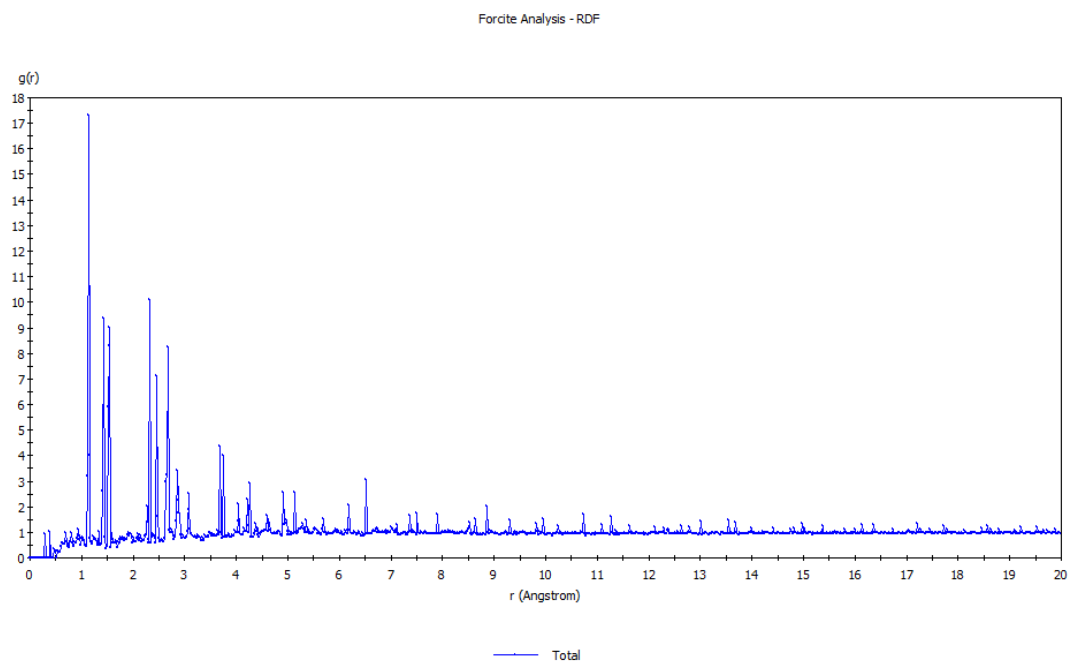

FIGURE 4.The computed part radial distribution functions(RDF) of intrinsic Graphene/PANI.

\section{CONCLUSION}

Formulate a theoretical reference method for the development of materials' property is important. In this study, DFT calculations were carried out to evaluate the electronic property of Graphene/PANI. Based on the calculated band gap, Graphene/PANI exhibits excellent conductivity. By comparing the rdf of materials, the Graphene/PANI exhibit excellent adsorption capacity towards small molecules. These results imply that the Graphene/PANI is more favorable in detecting gas because of its high sensitivity and adsorption. The results of this study may serve as a basis for the design of effective sensors for different gases. 


\section{ACKNOWLEDGMENTS}

This work was financially supported by National Natural Science Foundation of China (51675384).

\section{REFERENCES}

1. M. Ates, A review study of (bio)sensor systems based on conducting polymers, Materials science \& engineering. C, Materials for biological applications, 33 (2013) 1853-1859.

2. S. Das, V. Jayaraman, SnO2: A comprehensive review on structures and gas sensors, Progress in Materials Science, 66 (2014) 112-255.

3. G. Jimenez-Cadena, J. Riu, F.X. Rius, Gas sensors based on nanostructured materials, The Analyst, 132 (2007) 1083-1099.

4. M. Xu, T. Liang, M. Shi, H. Chen, Graphene-like two-dimensional materials, Chemical reviews, 113 (2013) 3766-3798.

5. S. Gupta Chatterjee, S. Chatterjee, A.K. Ray, A.K. Chakraborty, Graphene-metal oxide nanohybrids for toxic gas sensor: A review, Sensors and Actuators B: Chemical, 221 (2015) 1170-1181.

6. J. Xu, Y. Wang, S. Hu, Nanocomposites of graphene and graphene oxides: Synthesis, molecular functionalization and application in electrochemical sensors and biosensors. A review, Microchimica Acta, 184 (2016) 1-44.

7. V. Chabot, D. Higgins, A. Yu, X. Xiao, Z. Chen, J. Zhang, A review of graphene and graphene oxide sponge: material synthesis and applications to energy and the environment, Energy \& Environmental Science, 7 (2014) 1564.

8. Z. Wu, X. Chen, S. Zhu, Z. Zhou, Y. Yao, W. Quan, B. Liu, Enhanced sensitivity of ammonia sensor using graphene/polyaniline nanocomposite, Sensors and Actuators B: Chemical, 178 (2013) 485-493.

9. C.N.R. Rao, K. Gopalakrishnan, A. Govindaraj, Synthesis, properties and applications of graphene doped with boron, nitrogen and other elements, Nano Today, 9 (2014) 324-343.

10. H. Peng, J.P. Perdew, Rehabilitation of the Perdew-Burke-Ernzerhof generalized gradient approximation for layered materials, Physical Review B, 95 (2017). 